\title{
NONCOVARIANT GAUGES IN SIMPLE SUPERGRAVITY
}

\author{
GIAMPIERO ESPOSITO \\ Istituto Nazionale di Fisica Nucleare, Sezione di Napoli, \\ Mostra d'Oltremare Padiglione 20, 80125 Napoli, Italy; \\ Dipartimento di Scienze Fisiche, \\ Mostra d'Oltremare Padiglione 19, 80125 Napoli, Italy \\ GIUSEPPE POLLIFRONE \\ Dipartimento di Fisica, Università di Roma "La Sapienza" \\ and INFN, Sezione di Roma, Piazzale Aldo Moro 2, 00185 Roma, Italy
}

\begin{abstract}
A gauge-averaging functional of the axial type is studied for simple supergravity at one loop about flat Euclidean four-space bounded by a three-sphere, or two concentric three-spheres. This is a generalization of recent work on the axial gauge in quantum supergravity on manifolds with boundary. Ghost modes obey nonlocal boundary conditions of the spectral type, in that half of them obey Dirichlet or Neumann conditions at the boundary. In both cases, they give a vanishing contribution to the one-loop divergence. The admissibility of noncovariant gauges at the classical level is also proved.
\end{abstract}

PACS numbers: 04.65.+e, 98.80.Hw 


\section{Noncovariant Gauges in Simple Supergravity}

\section{Introduction}

The recent investigations of boundary conditions and heat-kernel asymptotics in Euclidean quantum gravity have led to new work on the application of noncovariant gauges in the quantization of Einstein's gravity and simple supergravity. ${ }^{1-3}$ The motivations of such an analysis are as follows.

(i) To improve the understanding of the one-loop semiclassical approximation. If quantum theory is viewed as a theory of small disturbances of the underlying classical theory, one should be able to compute at least the first quantum corrections in powers of $\hbar$, if such a perturbative scheme can be of any help (despite the well known lack of perturbative renormalizability).

(ii) To obtain a complete picture of admissible boundary conditions for the quantization of gauge fields and gravitation. Boundary conditions are here viewed as an essential element of any quantization scheme, as is suggested by recent progress in Euclidean quantum gravity. ${ }^{3,4}$

(iii) To ensure self-adjointness of the elliptic operators acting on graviton and gravitino perturbations, when a problem with boundary is studied. ${ }^{1-3}$

(iv) To understand whether supergravity theories are at least one-loop finite in the presence of boundaries. ${ }^{2,3}$

In simple supergravity, which is the object of our investigation, it is by now well known that one has a choice of local or nonlocal boundary conditions on gravitino perturbations. The former may involve complementary projection operators at the boundary, ${ }^{5}$ or may fix on the initial surface the whole primed part of tangential components of gravitino perturbations, and on the final surface the whole unprimed part of tangential components of gravitino perturbations. ${ }^{6,7}$ The latter rely instead on the following scheme. ${ }^{2,3}$ The massless Rarita-Schwinger potential subject to gauge conditions and linearized supersymmetry constraints is split into a regular part and a singular part. The regular part may be written as an infinite sum of modes multiplying harmonics having positive eigenvalues of the 


\section{Noncovariant Gauges in Simple Supergravity}

intrinsic three-dimensional Dirac operator of the boundary. The singular part is instead an infinite sum of modes multiplying harmonics having negative eigenvalues of the intrinsic three-dimensional Dirac operator of the boundary. One thus performs a nonlocal operation, i.e. the separation of the spectrum of a first-order elliptic operator into its positive and negative part. ${ }^{2,8}$ This is closely related to a positive- and negative-frequency split, which is suitable both for scattering theory and one-loop quantum cosmology. ${ }^{6,7}$

These nonlocal boundary conditions of the spectral type for gravitino perturbations can be written in the form ${ }^{2}$

$$
\begin{aligned}
& {\left[\psi_{i(+)}^{A}\right]_{\partial M}=0,} \\
& {\left[\widetilde{\psi}_{i(+)}^{A^{\prime}}\right]_{\partial M}=0,}
\end{aligned}
$$

where the label $(+)$ denotes the part of the perturbation potential corresponding to the regular part of the underlying classical theory. In Eqs. (1.1) and (1.2) one deals with the tangential components of the perturbation potential. Their expression in terms of spinor and tetrad fields is

$$
\begin{aligned}
\psi_{i}^{A} & =\Gamma^{C^{\prime}{ }_{B}} e^{B}{ }_{C^{\prime} i}, \\
\widetilde{\psi}_{i}^{A^{\prime}} & =\gamma^{C A_{B^{\prime}}^{\prime}} e^{B^{\prime}}{ }_{C i},
\end{aligned}
$$

where $\Gamma$ and $\gamma$ are the purely two-spinor part of Rarita-Schwinger potentials, while $e_{C^{\prime} i}^{B}$ is the spatial component of the two-spinor version of the tetrad. The infinitesimal gauge transformations for Rarita-Schwinger potentials are ${ }^{2}$

$$
\begin{gathered}
\widehat{\Gamma}_{B C}^{A^{\prime}} \equiv \Gamma_{B C}^{A^{\prime}}+\nabla_{B}^{A^{\prime}} \nu_{C}, \\
\widehat{\gamma}_{B^{\prime} C^{\prime}}^{A} \equiv \gamma_{B^{\prime} C^{\prime}}^{A}+\nabla_{B^{\prime}}^{A} \mu_{C^{\prime}} .
\end{gathered}
$$

To ensure invariance of the boundary conditions (1.1) and (1.2) under such gauge transformations, one begins by considering the conditions ${ }^{2}$

$$
\left[\nabla_{B}^{A^{\prime}} \nu_{C_{(+)}}\right]_{\partial M}=0
$$




$$
\begin{aligned}
& \text { Noncovariant Gauges in Simple Supergravity } \\
& \qquad\left[\nabla_{B^{\prime}}^{A} \mu_{C_{(+)}^{\prime}}\right]_{\partial M}=0 .
\end{aligned}
$$

In the (one-loop) quantum theory, the spinor fields $\nu_{C}$ and $\mu_{C^{\prime}}$ should be regarded as the ghost fields, and the boundary conditions (1.7a) and (1.8a) do not lead, by themselves, to a well-posed problem for ghost perturbations, since each of them leads to 8 conditions at the boundary. To overcome this problem, the work in Ref. 2 considered a particular subsector, obtained by contracting Eq. (1.7a) with the Euclidean normal $e_{e} n_{A^{\prime}}$, and Eq. (1.8a) with the Euclidean normal $e_{e} n_{A}^{B^{\prime}}$. As shown in Ref. 2, this prescription leads to a peculiar set of spectral boundary conditions on ghost modes, in that half of them (i.e. the regular ones) obey Neumann conditions at the boundary.

However, when gauge-averaging functionals of the axial type are chosen, another possibility consists in contracting Eqs. (1.7a) and (1.8a) with that particular linear combination of Euclidean normals which then sets to zero at the boundary the action of the ghost operators on $\nu^{C}$ and $\mu^{C^{\prime}}$, respectively. For example, if the axial-type gauge functionals are chosen as in Ref. 2:

$$
\begin{gathered}
\Phi^{A}(\Gamma) \equiv{ }_{e} n_{C C^{\prime}} \Gamma^{A C C^{\prime}}, \\
\widetilde{\Phi}^{A^{\prime}}(\gamma) \equiv{ }_{e} n_{C C^{\prime}} \gamma^{A^{\prime} C^{\prime} C},
\end{gathered}
$$

one finds the ghost operators ${ }^{2}$

$$
\begin{gathered}
\mathcal{D}_{C}{ }^{A} \equiv{ }_{e} n_{C C^{\prime}} \nabla^{A C^{\prime}}, \\
\mathcal{F}_{C^{\prime}}{ }^{A^{\prime}} \equiv{ }_{e} n_{C C^{\prime}} \nabla^{C A^{\prime}},
\end{gathered}
$$

which, of course, act linearly on $\nu^{C}$ and $\mu^{C^{\prime}}$, respectively. On the other hand, contraction of Eq. (1.7a) with ${ }_{e} n^{C}{ }^{\prime}$ leads to

$$
\left[\mathcal{D}_{C B} \nu_{(+)}^{C}\right]_{\partial M}=0 .
$$

An analogous procedure for Eq. (1.8a) leads to

$$
\left[\mathcal{F}_{C^{\prime} B^{\prime}} \mu_{(+)}^{C^{\prime}}\right]_{\partial M}=0 .
$$


At a deeper level, Eq. (1.13) is obtained by requiring that the boundary conditions

$$
\left[\Phi^{A}(\Gamma)\right]_{\partial M}=0
$$

should be invariant under the infinitesimal gauge transformations (1.5):

$$
\left[\Phi^{A}(\widehat{\Gamma})\right]_{\partial M}=0
$$

Similarly, Eq. (1.14) is obtained if one requires that the boundary conditions

$$
\left[\widetilde{\Phi}^{A^{\prime}}(\gamma)\right]_{\partial M}=0
$$

should be preserved by the infinitesimal gauge transformations (1.6):

$$
\left[\widetilde{\Phi}^{A^{\prime}}(\widehat{\gamma})\right]_{\partial M}=0
$$

Moreover, by virtue of the linear action of the ghost operators, Eqs. (1.13) and (1.14) are equivalent to spectral boundary conditions where all regular ghost modes are set to zero on the final surface, if the initial three-surface shrinks to a point (as it happens in the Hartle-Hawking approach to quantum cosmology $\left.{ }^{9}\right)$.

In our paper, we consider a more general class of gauge-averaging functionals of the axial type. They are defined as

$$
\begin{gathered}
\chi^{A}(\Gamma) \equiv{ }_{e} n_{C C^{\prime}} \Gamma^{(A C) C^{\prime}}, \\
\widetilde{\chi}^{A^{\prime}}(\gamma) \equiv{ }_{e} n_{C C^{\prime}} \gamma^{\left(A^{\prime} C^{\prime}\right) C},
\end{gathered}
$$

where round brackets denote symmetrization over spinor indices of the same kind. In Ref. 2 it was pointed out that the corresponding one-loop properties were still unknown and should have been analyzed. It has been therefore our aim to study a Faddeev-Popov path-integral representation for the one-loop wave function of the universe which involves a Gaussian average over the gauge functionals (1.17) and (1.18), in the limit of small threegeometry. ${ }^{10}$ The corresponding background can be taken to be a portion of flat Euclidean four-space bounded by a three-sphere of radius $a,{ }^{3,10}$ with radial coordinate $\tau \in[0, a]$. 


\section{Noncovariant Gauges in Simple Supergravity}

Section 2 derives ghost operators and boundary conditions when the gauge-averaging functionals (1.17) and (1.18) are chosen. One-loop properties are studied in Sec. 3, and the admissibility of our noncovariant gauges at the classical level is proved in Sec. 4 . Concluding remarks are presented in Sec. 5.

\section{Ghost Operators and Eigenvalue Equations}

In the Faddeev-Popov path integral for simple supergravity in the axial gauge, one has to perform a gaussian average involving the left-hand sides of (1.17) and (1.18), jointly with their corresponding ghost terms.

In the case of massless gravitino perturbations, as we said in the introduction, the ghost operators are obtained by studying the behaviour of (1.17) and (1.18) under the infinitesimal gauge transformations (1.5) and (1.6), respectively. Thus, the ghost operators acting on the spinor fields $\nu^{C}$ and $\mu^{C^{\prime}}$ read

$$
\mathcal{P}^{A}{ }_{C} \equiv\left({ }_{e} n_{C C^{\prime}} \nabla^{A C^{\prime}}-\varepsilon^{A}{ }_{C} e^{n_{F C^{\prime}}} \nabla^{F C^{\prime}}\right)
$$

and

$$
\mathcal{R}^{A^{\prime}}{ }_{C^{\prime}} \equiv\left({ }_{e} n_{C C^{\prime}} \nabla^{C A^{\prime}}-\varepsilon^{A^{\prime}}{ }_{C^{\prime}}{ }_{e} n_{C F^{\prime}} \nabla^{C F^{\prime}}\right)
$$

One can write the eigenfunction expansions for the spinor fields $\nu^{C}$ and $\mu^{C^{\prime}}$ as ${ }^{2}$

$$
\begin{aligned}
\nu^{C} & =\sum_{\lambda} \mathcal{B}_{\lambda}^{1} \nu_{(\lambda)}^{C}, \\
\mu^{C^{\prime}} & =\sum_{\tilde{\lambda}} \mathcal{B}_{\tilde{\lambda}}^{2} \mu_{(\tilde{\lambda})}^{C^{\prime}},
\end{aligned}
$$

and then analyze the following eigenvalue equations:

$$
\begin{gathered}
\mathcal{P}^{A}{ }_{C} \nu_{(\lambda)}^{C}=\lambda \nu_{(\lambda)}^{A}, \\
\mathcal{R}^{A^{\prime}}{ }_{C^{\prime}} \mu_{(\tilde{\lambda})}^{C^{\prime}}=\tilde{\lambda} \mu_{(\tilde{\lambda})}^{A^{\prime}} .
\end{gathered}
$$




\section{Noncovariant Gauges in Simple Supergravity}

It is well known that for massless gravitinos, expressed in the form (1.3) and (1.4) and subject to the gauge transformations (1.5) and (1.6), the background is forced to be Ricciflat. ${ }^{11}$ This happens since the spinor fields $\nu_{C}$ and $\mu_{C^{\prime}}$ have to be freely specifiable inside the background four-manifold. Considering furthermore a local description of RaritaSchwinger potentials occurring in (1.3) and (1.4) in terms of a second set of potentials, one finds that the background with boundary is further restricted to be totally flat. ${ }^{2,12}$

We here study a flat Euclidean background bounded by a three-sphere. Thus, bearing in mind that ${ }^{2}$

$$
{ }_{e} n_{A A^{\prime}} \nabla^{A A^{\prime}} \nu_{(\lambda)}^{C}=-\frac{\partial}{\partial \tau} \nu_{(\lambda)}^{C}
$$

the left-hand sides of Eqs. (2.5) and (2.6) take the form (cf. Ref. 2)

$$
\mathcal{P}^{A}{ }_{C} \nu_{(\lambda)}^{C}=-\left(3 \frac{\partial}{\partial \tau} \nu_{(\lambda)}^{A}+2 e_{e} n_{C^{\prime}}\left(C e^{A) C^{\prime} i(4)} \nabla_{i} \nu_{C(\lambda)}\right)\right.
$$

and

$$
\mathcal{R}^{A^{\prime}}{ }_{C^{\prime}} \mu_{(\tilde{\lambda})}^{C^{\prime}}=-\left(3 \frac{\partial}{\partial \tau} \mu_{(\tilde{\lambda})}^{A^{\prime}}+2{ }_{e} n_{C}{ }^{\left(C^{\prime}\right.} e^{\left.A^{\prime}\right) C i(4)} \nabla_{i} \mu_{C^{\prime}(\tilde{\lambda})}\right)
$$

respectively. Further details about the notation can be found in Ref. 2 .

One now has to impose boundary conditions for the solutions of the eigenvalue equations (2.5) and (2.6). A suitable set of boundary conditions consist in the vanishing of the axial-type gauge-averaging functionals (1.17) and (1.18) at the boundary. This choice can be viewed as the generalization of magnetic boundary conditions of Euclidean Maxwell theory. ${ }^{3}$ More precisely, for fields of spin $1, \frac{3}{2}$ and 2 one can always set to zero at the boundary the gauge-averaging functional (either covariant, e.g. Lorentz, harmonic or de Donder, or noncovariant, e.g. axial or Coulomb). This is part of a set of mixed boundary

conditions for the quantum theory. ${ }^{1-4}$ The requirement that such boundary conditions should be preserved under the infinitesimal gauge transformations (1.5) and (1.6) leads to

$$
\left[\mathcal{P}^{A}{ }_{C} \nu_{(+)}^{C}\right]_{\partial M}=0
$$

and

$$
\left[\mathcal{R}^{A^{\prime}} C^{\prime} \mu_{(+)}^{C^{\prime}}\right]_{\partial M}=0
$$




\section{Noncovariant Gauges in Simple Supergravity}

\section{One-loop Analysis in the Axial Gauge}

In this section we study the one-loop properties of our ghost fields in flat Euclidean background bounded by a three-sphere. For this purpose, we expand the ghost fields in harmonics on a family of three-spheres centred on the origin ${ }^{2}$

$$
\begin{aligned}
\nu^{A} & =\sum_{n=0}^{\infty} \sum_{p, q=1}^{(n+1)(n+2)} \alpha_{n}^{p q}\left[m_{n p}(\tau) \rho^{n q A}+\widetilde{r}_{n p}(\tau) \bar{\sigma}^{n q A}\right], \\
\mu^{A^{\prime}} & =\sum_{n=0}^{\infty} \sum_{p, q=1}^{(n+1)(n+2)} \alpha_{n}^{p q}\left[\widetilde{m}_{n p}(\tau) \bar{\rho}^{n q A^{\prime}}+r_{n p}(\tau) \sigma^{n q A^{\prime}}\right]
\end{aligned}
$$

where the $\alpha_{n}$ are block-diagonal matrices with blocks $\left(\begin{array}{cc}1 & 1 \\ 1 & -1\end{array}\right)$, and the harmonics obey the eigenvalue equations described, for example, in Ref. 2. Hence one finds that the eigenvalue equations (2.5) and (2.6) for the ghost modes occurring in the expansions (3.1) and (3.2) read

$$
\begin{gathered}
-\left(3 \frac{d}{d \tau}+\frac{n}{\tau}\right) m_{n p}=\lambda_{n} m_{n p} \\
-\left(3 \frac{d}{d \tau}-\frac{(n+3)}{\tau}\right) \widetilde{r}_{n p}=\lambda_{n} \widetilde{r}_{n p} \\
-\left(3 \frac{d}{d \tau}-\frac{n}{\tau}\right) \widetilde{m}_{n p}=\widetilde{\lambda}_{n} \widetilde{m}_{n p} \\
-\left(3 \frac{d}{d \tau}+\frac{(n+3)}{\tau}\right) r_{n p}=\widetilde{\lambda}_{n} r_{n p}
\end{gathered}
$$

The solutions of Eqs. (3.3)-(3.6) are (cf. Eqs. (2.21)-(2.24) in Ref. 2)

$$
\begin{aligned}
& m_{n p}\left(\tau, \lambda_{n}\right)=\tau^{-\frac{n}{3}} e^{-\frac{\lambda_{n}}{3} \tau} m_{n p}^{0}, \\
& \widetilde{r}_{n p}\left(\tau, \lambda_{n}\right)=\tau^{\frac{(n+3)}{3}} e^{-\frac{\lambda_{n}}{3} \tau} \widetilde{r}_{n p}^{0}, \\
& \widetilde{m}_{n p}\left(\tau, \widetilde{\lambda}_{n}\right)=\tau^{\frac{n}{3}} e^{-\frac{\widetilde{\lambda}_{n}}{3} \tau} \widetilde{m}_{n p}^{0},
\end{aligned}
$$




\section{Noncovariant Gauges in Simple Supergravity}

$$
r_{n p}\left(\tau, \widetilde{\lambda}_{n}\right)=\tau^{-\frac{(n+3)}{3}} e^{-\frac{\widetilde{\lambda}_{n}}{3} \tau} r_{n p}^{0},
$$

where $m_{n p}^{0}, \widetilde{r}_{n p}^{0}, \widetilde{m}_{n p}^{0}$ and $r_{n p}^{0}$ denote some multiplicative constants which are determined by the boundary values. Remarkably, regularity at the origin is guaranteed by the vanishing of the $m_{n p}$ and $r_{n p}$ modes, for all $\tau \in[0, a]$, where $a$ is the radius of the three-sphere boundary. Moreover, one has to impose the boundary conditions (2.10) and (2.11) on the remaining (regular) set of ghost modes. Since the ghost operators act linearly on ghost modes (see (2.5) and (2.6)), such spectral boundary conditions set to zero everywhere the $\widetilde{m}_{n p}$ and $\widetilde{r}_{n p}$ modes. Hence the whole set of ghost modes are forced to vanish, with this version of the boundary conditions in the axial gauge.

By contrast, the spectral boundary conditions studied in Ref. 2 and mentioned in the introduction:

$$
\begin{gathered}
{\left[{ }_{e} n^{B}{ }_{A^{\prime}} \nabla^{A^{\prime}}{ }_{B} \nu_{C_{(+)}}\right]_{\partial M}=0,} \\
{\left[{ }_{e} n_{A}{ }^{B^{\prime}} \nabla_{B^{\prime}}^{A} \mu_{C^{\prime}(+)}\right]_{\partial M}=0,}
\end{gathered}
$$

lead to Neumann boundary conditions at the boundary, i.e.

$$
\left[\frac{d}{d \tau} \widetilde{r}_{n p}\right]_{\tau=a}=0
$$

and

$$
\left[\frac{d}{d \tau} \widetilde{m}_{n p}\right]_{\tau=a}=0 .
$$

One thus gets, from (3.8) and (3.9), the same discrete spectra for the regular modes found in Ref. 2, although the axial gauge-averaging functionals (1.9) and (1.10) differ from (1.17) and (1.18) considered in our paper. Such discrete spectra are

$$
\begin{gathered}
\lambda_{n}=\frac{(n+3)}{a} \quad \forall n \geq 0, \\
\tilde{\lambda}_{n}=\frac{n}{a} \quad \forall n \geq 0 .
\end{gathered}
$$




\section{Noncovariant Gauges in Simple Supergravity}

Setting for convenience $a=1$, one can define the following $\zeta$-function for a first-order, elliptic and positive-definite operator $\mathcal{A}$ (cf. (2.8)) with spectrum (3.13):

$$
\zeta_{\mathcal{A}}(s) \equiv \sum_{n=0}^{\infty}(n+1)(n+2)(n+3)^{-s} .
$$

Moreover, a first-order, elliptic and nonnegative operator $\mathcal{B}$ exists (cf. (2.9)), with spectrum (3.14) and finite-dimensional null-space, and its $\zeta$-function can be defined as

$$
\zeta_{\mathcal{B}}(s) \equiv 2+\sum_{n=1}^{\infty}(n+1)(n+2) n^{-s}
$$

where the dimension of the null-space has been included in the definition of the $\zeta$-function, following Ref. 2. One then finds (here, $\zeta_{H}$ and $\zeta_{R}$ are the Hurwitz and Riemann $\zeta$ functions, respectively, defined in the appendix of Ref. 2)

$$
\begin{gathered}
\zeta_{\mathcal{A}}(0)=\zeta_{H}(-2,3)-3 \zeta_{H}(-1,3)+2 \zeta_{H}(0,3)=-\frac{3}{4} \\
\zeta_{\mathcal{B}}(0)=2+\zeta_{R}(-2)+3 \zeta_{R}(-1)+2 \zeta_{R}(0)=\frac{3}{4} .
\end{gathered}
$$

Hence the ghost gravitino contribution to the one-loop divergence, in both versions of the axial gauge, vanishes in our flat background bounded by a three-sphere:

$$
\zeta_{\text {ghost }}(0)=\zeta_{\mathcal{A}}(0)+\zeta_{\mathcal{B}}(0)=0 .
$$

Note that no Nielsen-Kallosh ghost fields ${ }^{13,14}$ occur in our calculation, since, as explained in Ref. 2, the axial gauge has already the effect to reduce the linearized gravitino potential to its two physical degrees of freedom, corresponding to helicities $\frac{3}{2}$ and $-\frac{3}{2}$.

So far, motivated by quantum cosmology, we have studied backgrounds where the initial three-surface shrinks to zero. However, in quantum field theory one deals with a path-integral representation of transition amplitudes with suitable data on two boundary three-surfaces. Hence we now analyze ghost modes of simple supergravity on a flat Euclidean four-manifold bounded by two concentric three-spheres. The form of ghost modes 


\section{Noncovariant Gauges in Simple Supergravity}

is again (3.7)-(3.10), and the spectral boundary conditions resulting from (1.7b) and (1.8b) are

$$
\begin{aligned}
& {\left[\frac{d}{d \tau} m_{n p}\right]_{\tau=\tau_{-}}=0,} \\
& {\left[\frac{d}{d \tau} r_{n p}\right]_{\tau=\tau_{-}}=0,} \\
& {\left[\frac{d}{d \tau} \widetilde{m}_{n p}\right]_{\tau=\tau_{+}}=0,} \\
& {\left[\frac{d}{d \tau} \widetilde{r}_{n p}\right]_{\tau=\tau_{+}}=0,}
\end{aligned}
$$

where $\tau_{-}$and $\tau_{+}$are the three-sphere radii $\left(\tau_{+}>\tau_{-}\right)$. Similarly to the analysis of Ref. 2 , such boundary conditions lead to incompatible solutions for the eigenvalues $\lambda_{n}$ and $\widetilde{\lambda}_{n}$. In particular, (3.20) and (3.23) imply that $\lambda_{n}=-\frac{n}{\tau_{-}} \leq 0$ and $\lambda_{n}=\frac{(n+3)}{\tau_{+}}>0$, while (3.21) and (3.22) lead to $\tilde{\lambda}_{n}=-\frac{(n+3)}{\tau_{-}}<0$ and $\tilde{\lambda}_{n}=\frac{n}{\tau_{+}} \geq 0$. Hence no nontrivial ghost modes exist. This remains true if one uses instead the spectral boundary conditions (2.10) and (2.11) when $\tau=\tau_{-}$and $\tau=\tau_{+}$, by virtue of (2.5), (2.6) and (3.7)-(3.10).

Following Refs. 1,2, the one-loop divergence for simple supergravity in the axial gauge reduces therefore to the contribution resulting from three-dimensional transverse-traceless perturbations for gravitons and gravitinos:

$$
\zeta(0)=-\frac{278}{45}+\frac{289}{360}=-\frac{43}{8}
$$

when only one bounding three-sphere exists, and

$$
\zeta(0)=-5
$$

when two concentric three-sphere boundaries occur. 


\section{Noncovariant Gauges in Simple Supergravity}

\section{Classical Admissibility of Axial Gauges}

The axial gauge-averaging functionals play an important role in the path-integral approach to quantum gravity, since they lead to self-adjoint operators on metric and gravitino perturbations, and are part of the quantization programme in noncovariant gauges. ${ }^{15-20}$ This makes it interesting to investigate the classical counterpart of the axial gauge-averaging functionals (1.17) and (1.18). Hence we impose, in the underlying classical theory, the axial gauge, i.e.

$$
\begin{gathered}
e^{n_{C C^{\prime}}} \Gamma^{(A C) C^{\prime}}=0, \\
e^{n_{C C^{\prime}}} \gamma^{\left(A^{\prime} C^{\prime}\right) C} .
\end{gathered}
$$

The preservation of the gauge conditions (4.1) and (4.2) under the infinitesimal gauge transformations (1.5) and (1.6) leads to the following differential equations for the spinor fields $\nu^{A}$ and $\mu^{A^{\prime}}$ :

$$
\begin{aligned}
& { }_{e} n_{C C^{\prime}} \nabla^{C^{\prime}\left(A_{\nu} C\right)}=0, \\
& { }_{e} n_{C C^{\prime}} \nabla^{C\left(A^{\prime}\right.} \mu^{\left.C^{\prime}\right)}=0 .
\end{aligned}
$$

Hence the spinor fields $\nu^{A}$ and $\mu^{A^{\prime}}$ are no longer freely specifiable, but they have to satisfy Eqs. (4.3) and (4.4). Interestingly, a sufficient condition for the validity of such equations is obtained if $\nu^{A}$ and $\mu^{A^{\prime}}$ are solutions of the twistor equation. ${ }^{12}$

The restriction on gauge fields resulting from the preservation of gauge conditions should not be surprising, and is indeed a familiar property in field theory. For example, it is well known that (Euclidean) Maxwell theory is invariant under the following infinitesimal gauge transformations:

$$
{ }^{f} A_{b}=A_{b}+\nabla_{b} f
$$

where $A_{b}$ is the electromagnetic potential and the function $f$ is freely specifiable. However, after imposing a gauge condition, the function $f$ has to obey a differential equation, instead of being freely specifiable. On choosing the axial gauge:

$$
n^{b} A_{b}=0
$$




\section{Noncovariant Gauges in Simple Supergravity}

it is possible to ensure that the gauge-transformed potential (4.5) does actually obey the gauge (4.6), provided that $n^{b} \nabla_{b} f=0$. In the case of the Lorentz gauge:

$$
\nabla^{b} A_{b}=0
$$

the preservation of Eq. (4.7) under (4.5) forces the function $f$ to obey the four-dimensional Laplace equation, if the background is Riemannian. Another choice is the Coulomb gauge, i.e.

$$
{ }^{(3)} \nabla^{i} A_{i}=0
$$

where ${ }^{(3)} \nabla^{i}$ is the three-dimensional tangential covariant derivative with respect to the Levi-Civita connection of the induced metric on the boundary. On requiring that the tangential components ${ }^{f} A_{i}$ of the gauge-transformed potential should satisfy Eq. (4.8), one finds that the function $f$ has to be a harmonic function on the boundary. Thus, provided that a gauge condition is imposed, and such a gauge condition also holds for the potential ${ }^{f} A_{b}$, the function $f$ is no longer freely specifiable, just as in the case of the axial gauge for the Rarita-Schwinger potential.

Now, it is convenient to study a mode-by-mode form of Eqs. (4.3) and (4.4), by using the expansions (3.1) and (3.2). Hence one finds (cf. Eqs. (3.3)-(3.6))

$$
\begin{gathered}
\left(3 \frac{d}{d \tau}+\frac{n}{\tau}\right) m_{n p}=0, \\
\left(3 \frac{d}{d \tau}-\frac{(n+3)}{\tau}\right) \widetilde{r}_{n p}=0, \\
\left(3 \frac{d}{d \tau}-\frac{n}{\tau}\right) \widetilde{m}_{n p}=0, \\
\left(3 \frac{d}{d \tau}+\frac{(n+3)}{\tau}\right) r_{n p}=0 .
\end{gathered}
$$

The solutions of Eqs. (4.9)-(4.12) can be written as

$$
\begin{aligned}
& m_{n p}(\tau)=\tau^{-\frac{n}{3}} m_{n p}^{0}, \\
& \widetilde{r}_{n p}(\tau)=\tau^{\frac{(n+3)}{3}} \widetilde{r}_{n p}^{0},
\end{aligned}
$$


Noncovariant Gauges in Simple Supergravity

$$
\begin{gathered}
\widetilde{m}_{n p}(\tau)=\tau^{\frac{n}{3}} \widetilde{m}_{n p}^{0}, \\
r_{n p}(\tau)=\tau^{-\frac{(n+3)}{3}} r_{n p}^{0} .
\end{gathered}
$$

To ensure regularity at the origin it is necessary to set to zero everywhere the modes $m_{n p}$ and $r_{n p}$. Hence we have proved that the axial gauge is admissible at the classical level, provided that the irregular modes occurring in the expansions in harmonics (3.1) and (3.2) vanish for all $\tau \in[0, a]$. As far as the regular modes are concerned, one can point out that Eqs. (4.3) and (4.4) should hold everywhere, and hence, in particular, at the boundary. This, however, does not fix $\widetilde{r}_{n p}$ and $\widetilde{m}_{n p}$.

\section{Concluding Remarks}

The contributions of our paper are as follows.

(i) It has been shown that any choice of axial gauge-averaging functional is compatible with having the standard form of spectral boundary conditions on ghost modes, i.e. when half of them are set to zero at the boundary. The work in Ref. 2 only considered the Neumann option for such modes.

(ii) The most general form of the ghost operators resulting from a gauge-averaging functional of the axial type has been obtained in Eqs. (2.1) and (2.2). The corresponding ghost contribution to the one-loop divergence vanishes. Thus, all gauges of the axial type are equivalent in the quantum theory.

(iii) The admissibility of the axial gauge for problems with boundary has been proved at the classical level. One starts from a configuration where the axial gauge is fulfilled. One then performs a gauge transformation on the Rarita-Schwinger potentials. This restricts the class of spinor fields $\nu_{C}$ and $\mu_{C^{\prime}}$ occurring in Eqs. (1.5) and (1.6). They are no longer freely specifiable. They have instead to solve Eqs. (4.3) and (4.4). The mode-by-mode form of such equations has been given and solved in the local coordinates appropriate for the case when a portion of flat Euclidean four-space is bounded by a three-sphere.

Our investigations are part of a more general programme, devoted to the study of Euclidean quantum gravity and quantum supergravity in covariant and noncovariant gauges 


\section{Noncovariant Gauges in Simple Supergravity}

(cf. Refs. 15-20), when boundary effects are included. ${ }^{1-3}$ As shown in Refs. 3-5, there is increasing evidence that this programme is going to shed new light on the various approaches to the quantization of gauge theories, and on the fertile interplay between spectral geometry and quantum field theory.

As far as supergravity theories are concerned, a further line of development lies in the application to supersymmetric quantum cosmology. So far, the main emphasis has been on the application of Hamiltonian methods in such a branch of modern quantum cosmology (see Refs. 6,21 and references therein). It now remains to be seen whether the techniques applied in Refs. 1-3 and in our paper can improve the current understanding of the quantum state of the universe within a supersymmetric framework.

\section{Acknowledgments}

We are indebted to Ivan Avramidi and Alexander Kamenshchik for scientific collaboration on topics related to the one studied in our paper.

\section{References}

1. I. G. Avramidi, G. Esposito and A. Yu. Kamenshchik, Class. Quantum Grav. 13, 2361 (1996).

2. G. Esposito and A. Yu. Kamenshchik, Phys. Rev. D54, 3869 (1996).

3. G. Esposito, A. Yu. Kamenshchik and G. Pollifrone, Euclidean Quantum Gravity on Manifolds with Boundary, Fundamental Theories of Physics (Kluwer, Dordrecht, 1997).

4. I. G. Moss and P. J. Silva, Phys. Rev. D55, 1072 (1996).

5. H. C. Luckock, J. Math. Phys. 32, 1755 (1991).

6. P. D. D'Eath, Supersymmetric Quantum Cosmology (Cambridge University Press, Cambridge, 1996).

7. G. Esposito, Phys. Lett. B389, 510 (1996).

8. M. F. Atiyah, V. K. Patodi and I. M. Singer, Math. Proc. Camb. Phil. Soc. 77, 43 (1975).

9. J. B. Hartle and S. W. Hawking, Phys. Rev. D28, 2960 (1983). 
10. K. Schleich, Phys. Rev. D32, 1889 (1985).

11. S. Deser and B. Zumino, Phys. Lett. B29, 335 (1976).

12. G. Esposito, G. Gionti, A. Yu. Kamenshchik, I. V. Mishakov and G. Pollifrone, Int. J. Mod. Phys. D4, 735 (1995).

13. N. K. Nielsen Nucl. Phys. B140, 499 (1978).

14. R. E. Kallosh Nucl. Phys. B141, 141 (1978).

15. T. Matsuki, Phys. Rev. D19, 2879 (1979).

16. T. Matsuki, Phys. Rev. D21, 899 (1980).

17. D. M. Capper and G. Leibbrandt, Phys. Rev. D25, 1009 (1982).

18. D. M. Capper and G. Leibbrandt, Phys. Rev. D25, 2211 (1982).

19. T. Matsuki, Phys. Rev. D32, 3164 (1985).

20. G. Leibbrandt, Rev. Mod. Phys. 59, 1067 (1987).

21. P. Moniz, Int. J. Mod. Phys. A11, 4321 (1996). 